\title{
Evaluation of a new Implicit Coupling Algorithm for the Partitioned Fluid-Structure Interaction Simulation of Bileaflet Mechanical Heart Valves
}

\author{
Sebastiaan Annerel ${ }^{1}$, Joris Degroote ${ }^{1}$, Tom Claessens ${ }^{2}$, Jan Vierendeels ${ }^{1}$ \\ ${ }^{1}$ Department of Flow, Heat and Combustion Mechanics, Ghent University, \\ Sint-Pietersnieuwstraat 41, B-9000 Ghent, Belgium \\ ${ }^{2}$ BioMech, Department of Mechanics, University College Ghent, \\ Schoonmeersstraat 52, B-9000 Ghent, Belgium \\ E-mail: Sebastiaan.Annerel@UGent.be
}

\begin{abstract}
We present a newly developed Fluid-Structure Interaction coupling algorithm to simulate Bileaflet Mechanical Heart Valves dynamics in a partitioned way. The coupling iterations between the flow solver and the leaflet motion solver are accelerated by using the Jacobian with the derivatives of the pressure and viscous moments acting on the leaflets with respect to the leaflet acceleration. This Jacobian is used in the leaflet motion solver when new positions of the leaflets are computed during the coupling iterations. The Jacobian is numerically derived from the flow solver by applying leaflet perturbations. Instead of calculating this Jacobian every time step, the Jacobian is extrapolated from previous time steps and a recalculation of the Jacobian is only done when needed. The efficiency of our new algorithm is subsequently compared to existing algorithms which use fixed relaxation and dynamic Aitken $\Delta^{2}$ relaxation in the coupling iterations when the new positions of the leaflets are computed. Results show that dynamic Aitken $\Delta^{2}$ relaxation outperforms fixed relaxation. Moreover, during the opening phase of the valve, our new algorithm needs fewer subiterations per time step to achieve convergence than the method with Aitken $\Delta^{2}$ relaxation. Thus, our newly developed FSI coupling scheme outperforms the existing coupling schemes.
\end{abstract}

\section{Introduction}

Bileaflet Mechanical Heart Valves (BMHVs) are widely used and preferred for valve replacement because of their long life span and durability. However, current BMHV designs induce calcification and thromboembolism, which is believed to be due to non-physiological flow and turbulence generated by the valve leaflets [1]. Numerical flow simulations can thus provide relevant information for valve design optimization.

Numerical simulation of such a BMHV is a complex Fluid-Structure Interaction (FSI) problem. The movement of the leaflets strongly interacts with the surrounding fluid motion and therefore it needs to be computed through a coupling scheme. A description of FSI methods for BMHVs can be found in [1]. Unfortunately, when using separate solvers, not every FSI coupling scheme converges efficiently. In literature, the instability of explicit coupling schemes for the case of BMHVs was explained [2,3]. Thus, implicitness is necessary for strong coupling, which implies the introduction of a subiteration loop within each time step. However, for doing so, stable and efficient approximations of the subsequent angular accelerations of the leaflets are needed in each subiteration $k$. 
In this paper, we present a new coupling algorithm, which predicts the moments (and thus the angular accelerations) for the next coupling iteration through a linearization of Newton's Second Law with a numerically computed Jacobian. This method was first introduced in $[2,4]$ for one leaflet, but now extended to two leaflets. The components of the Jacobian are composed of the gradients of the moments (exerted by the flow on the leaflets) with respect to changes in leaflet angular accelerations. Hence, this Jacobian is numerically derived from our flow solver by perturbations of the leaflet positions. Subsequently, the algorithm is extended to perform a correct leaflet movement to and from the closed and opened position. Finally, the convergence process is accelerated by the use of an adaptive time step size and extrapolation techniques.

The paper is organized as follows. First, we derive the algorithm and analyze its implementation in a commercially available black box flow solver. Secondly, we test our algorithm on a 3D case of a BMHV. Subsequently, we discuss the characteristics of existing coupling schemes. Finally, we test each of the discussed schemes for the 3D BMHV case, which allows a comparison of their convergence stability and efficiency through numerical experiments. We conclude that our newly developed FSI algorithm for the partitioned simulation of a BMHV is stable and outperforms the existing coupling schemes in needed subiterations per time step.

\section{FSI Algorithm}

The flow diagram of the algorithm is visualized in figure 1 and will be explained hereafter. The implementation in FLUENT is done by journal files and user-defined functions (UDFs). For each subiteration, the journal file updates the mesh, solves the Navier-Stokes equations and reads the data file of the time step. Thus, this journal file solves the flow problem. The UDFs, on the other hand, simulate the structural part. They perform calculations with the obtained flow solution in order to check for convergence and to set the motion parameters for the next subiteration. They interact with and steer the actions of the journal file. In the following, the steps of the flow diagram are explained and discussed in detail.

\subsection{Valve mechanics: leaflet motion solver}

A BMHV can be modelled as a rigid casing in which two separate rigid leaflets rotate around their axes. Because the position of each rigid leaflet is solely determined by its opening angle, the valve has two degrees of freedom.

The movement of a rigid leaflet is governed by Newton's Second Law, i.e. the equilibrium between the angular momentum about its hinge and the product of its moment of inertia and its angular acceleration. For two leaflets, this gives the following two equations with $M_{i}, I_{i}$ and $\ddot{\theta}_{i}$ representing, respectively, the angular momentum, the moment of inertia and the angular acceleration of leaflet $i$ about its rotation axis:

$$
\left\{\begin{array}{l}
M_{1}=I_{1} \cdot \ddot{\theta}_{1} \\
M_{2}=I_{2} \cdot \ddot{\theta}_{2}
\end{array}\right.
$$

When the hinges are modelled as frictionless, the angular momentum acting on the leaflet is the pressure (and viscous) momentum exerted by the flow.

As mentioned above, an implicit algorithm is necessary for strong coupling with separate solvers. Within the subiteration process of each time step, implicitness between the subsequent subiterations is needed to obtain fast convergence. Thus, while taking into account the mutual interaction between the leaflets, equation (1) needs to be linearized for each subiteration $k+1$ of time step $n+1$ :

$$
\left\{\begin{array}{l}
M_{1}^{n+1, k}+\frac{\partial M_{1}}{\partial \ddot{\theta}_{1}}\left(\ddot{\theta}_{1}^{n+1, k+1}-\ddot{\theta}_{1}^{n+1, k}\right)+\frac{\partial M_{1}}{\partial \ddot{\theta}_{2}}\left(\ddot{\theta}_{2}^{n+1, k+1}-\ddot{\theta}_{2}^{n+1, k}\right)=I_{1} \cdot \ddot{\theta}_{1}^{n+1, k+1} \\
M_{2}^{n+1, k}+\frac{\partial M_{2}}{\partial \ddot{\theta}_{1}}\left(\ddot{\theta}_{1}^{n+1, k+1}-\ddot{\theta}_{1}^{n+1, k}\right)+\frac{\partial M_{2}}{\partial \ddot{\theta}_{2}}\left(\ddot{\theta}_{2}^{n+1, k+1}-\ddot{\theta}_{2}^{n+1, k}\right)=I_{2} \cdot \ddot{\theta}_{2}^{n+1, k+1}
\end{array}\right.
$$




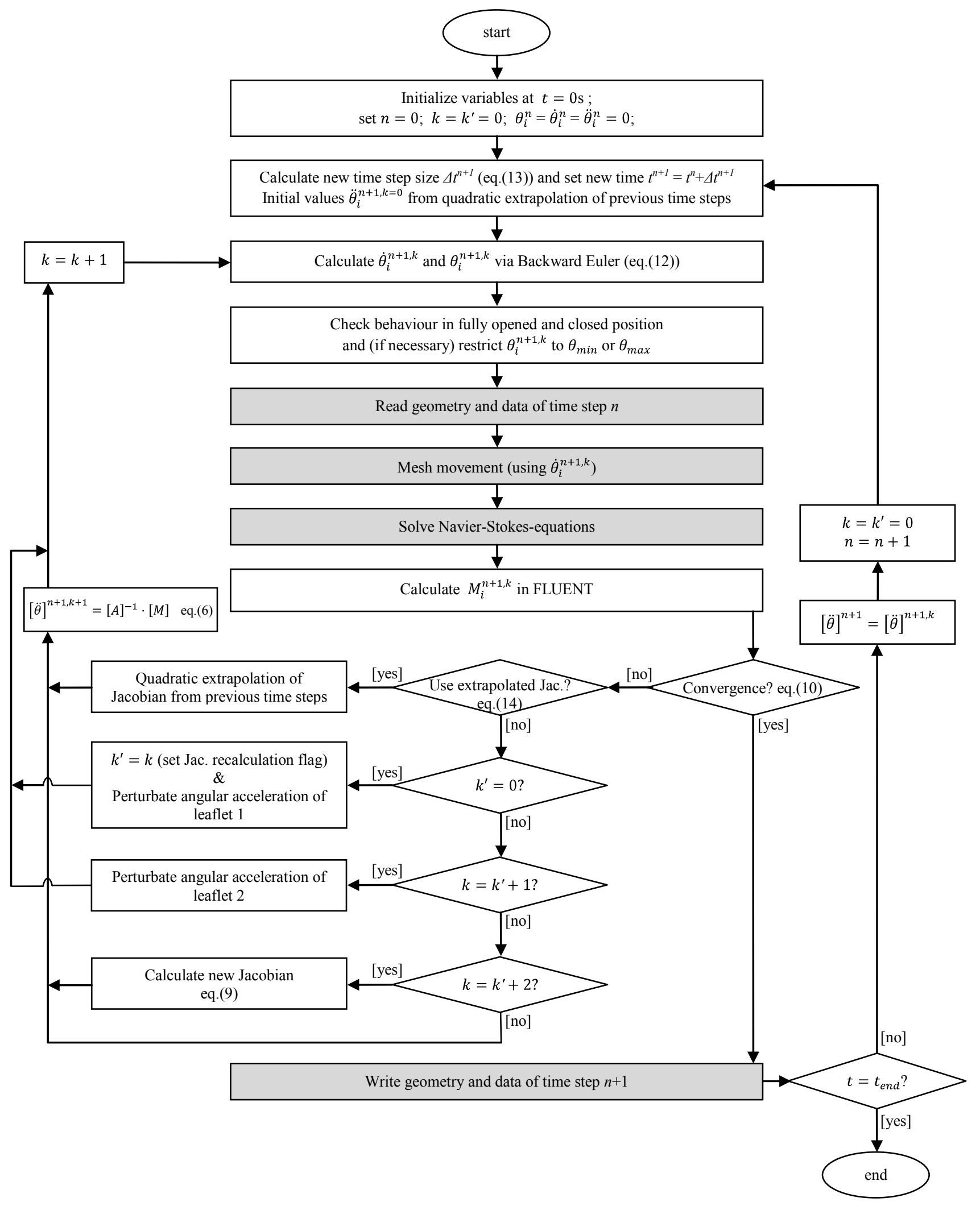

Figure 1. Flow diagram of implicit FSI coupling algorithm with two degrees of freedom. $n=$ time step, $k=$ subiteration step, $k^{\prime}=$ Jacobian recalculation flag, $i=$ leaflet number. Shaded: executed using generated journal file. Unshaded: executed using compiled UDFs. 
These equations can be rearranged as follows:

$$
\left\{\begin{array}{l}
M_{1}^{n+1, k}-\frac{\partial M_{1}}{\partial \ddot{\theta}_{1}} \ddot{\theta}_{1}^{n+1, k}-\frac{\partial M_{1}}{\partial \ddot{\theta}_{2}} \ddot{\theta}_{2}^{n+1, k}=I_{1} \cdot \ddot{\theta}_{1}^{n+1, k+1}-\frac{\partial M_{1}}{\partial \ddot{\theta}_{1}} \ddot{\theta}_{1}^{n+1, k+1}-\frac{\partial M_{1}}{\partial \ddot{\theta}_{2}} \ddot{\theta}_{2}^{n+1, k+1} \\
M_{2}^{n+1, k}-\frac{\partial M_{2}}{\partial \ddot{\theta}_{1}} \ddot{\theta}_{1}^{n+1, k}-\frac{\partial M_{2}}{\partial \ddot{\theta}_{2}} \ddot{\theta}_{2}^{n+1, k}=I_{2} \cdot \ddot{\theta}_{2}^{n+1, k+1}-\frac{\partial M_{2}}{\partial \ddot{\theta}_{1}} \ddot{\theta}_{1}^{n+1, k+1}-\frac{\partial M_{2}}{\partial \ddot{\theta}_{2}} \ddot{\theta}_{2}^{n+1, k+1}
\end{array}\right.
$$

This can be written in matrix notation:

or

$$
\left[\begin{array}{c}
M_{1}^{n+1, k}-\frac{\partial M_{1}}{\partial \ddot{\theta}_{1}} \ddot{\theta}_{1}^{n+1, k}-\frac{\partial M_{1}}{\partial \ddot{\theta}_{2}} \ddot{\theta}_{2}^{n+1, k} \\
M_{2}^{n+1, k}-\frac{\partial M_{2}}{\partial \ddot{\theta}_{1}} \ddot{\theta}_{1}^{n+1, k}-\frac{\partial M_{2}}{\partial \ddot{\theta}_{2}} \ddot{\theta}_{2}^{n+1, k}
\end{array}\right]=\left[\begin{array}{cc}
I_{1}-\frac{\partial M_{1}}{\partial \ddot{\theta}_{1}} & -\frac{\partial M_{1}}{\partial \ddot{\theta}_{2}} \\
-\frac{\partial M_{2}}{\partial \ddot{\theta}_{1}} & I_{2}-\frac{\partial M_{2}}{\partial \ddot{\theta}_{2}}
\end{array}\right] \cdot\left[\begin{array}{c}
\ddot{\theta}_{1}^{n+1, k+1} \\
\ddot{\theta}_{2}^{n+1, k+1}
\end{array}\right]
$$

$$
[M]^{n+1, k}=[A] \cdot[\ddot{\theta}]^{n+1, k+1}
$$

Thus, the matrix $[\ddot{\theta}]^{n+1, k+1}$ contains the angular accelerations on time step $n+1$ and subiteration $k+1$. The matrix $[A]$ is composed of the moments of inertia and the derivatives of the moments with respect to angular acceleration (i.e. the Jacobian). Finally, the matrix $[M]^{n+1, k}$ consists of all the remaining factors (in time step $n+1$ and subiteration $k$ ). For a well-posed problem, the matrix $[A]$ is nonsingular. So the previous equation can be rewritten as:

$$
[\ddot{\theta}]^{n+1, k+1}=[A]^{-1} \cdot[M]^{n+1, k}
$$

\subsection{Estimating the Jacobian and calculating the leaflet motions}

In order to solve equation (6) and to calculate the angular accelerations of the new subiteration, the Jacobian has to be known. However, when a black box flow solver is used, these derivatives are unknown, but can be approximated by finite differences. Therefore, three subiterations are needed within each time step. The first recalculation subiteration is the reference. We denote this subiteration by setting $k$ ' $=k$, i.e. setting the "Jacobian recalculation flag". In the second and third recalculation subiteration $\left(k=k^{\prime}+1\right.$ and $\left.k=k^{\prime}+2\right)$, a small perturbation $\delta$ is subsequently induced to the angular acceleration of each of the leaflets, while keeping the other one fixed. The value of this perturbation parameter $\delta$ ranges between a minimal value (in order to obtain a significant difference between the "reference moment" and the "perturbation moment") and a maximal value (above which the mesh motion could fail). In [2], it was shown that the exact choice of this parameter is not very sensitive. For each Jacobian recalculation subiteration, the moments acting on the leaflets are calculated and stored. This can be summarized as follows:

$$
\begin{gathered}
k=k^{\prime} \Rightarrow\left\{\begin{array} { l } 
{ \ddot { \theta } _ { 1 } ^ { n + 1 , k = k ^ { \prime } } } \\
{ \ddot { \theta } _ { 2 } ^ { n + 1 , k = k ^ { \prime } } }
\end{array} \quad \Rightarrow \left\{\begin{array}{l}
M_{1}^{n+1, k=k^{\prime}} \\
M_{2}^{n+1, k=k^{\prime}}
\end{array}\right.\right. \\
k=k^{\prime}+1 \Rightarrow\left\{\begin{array} { l } 
{ \ddot { \theta } _ { 1 } ^ { n + 1 , k = k ^ { \prime } + 1 } = \ddot { \theta } _ { 1 } ^ { n + 1 , k = k ^ { \prime } } + \delta } \\
{ \ddot { \theta } _ { 2 } ^ { n + 1 , k = k ^ { \prime } + 1 } = \ddot { \theta } _ { 2 } ^ { n + 1 , k = k ^ { \prime } } }
\end{array} \Rightarrow \left\{\begin{array}{l}
M_{1}^{n+1, k=k^{\prime}+1} \\
M_{2}^{n+1, k=k^{\prime}+1}
\end{array}\right.\right. \\
k=k^{\prime}+2 \Rightarrow\left\{\begin{array} { l } 
{ \ddot { \theta } _ { 1 } ^ { n + 1 , k = k ^ { \prime } + 2 } = \ddot { \theta } _ { 1 } ^ { n + 1 , k = k ^ { \prime } } } \\
{ \ddot { \theta } _ { 2 } ^ { n + 1 , k = k ^ { \prime } + 2 } = \ddot { \theta } _ { 2 } ^ { n + 1 , k = k ^ { \prime } } + \delta }
\end{array} \Rightarrow \left\{\begin{array}{l}
M_{1}^{n+1, k=k^{\prime}+2} \\
M_{2}^{n+1, k=k^{\prime}+2}
\end{array}\right.\right.
\end{gathered}
$$

The derivatives are estimated using finite differences $(i, j=1,2)$ :

$$
\frac{\partial M_{i}}{\partial \ddot{\theta}_{j}} \approx \frac{\Delta M_{i}}{\Delta \ddot{\theta}_{j}}
$$


This gives for each component of the Jacobian:

$$
\begin{aligned}
& \frac{\partial M_{1}}{\partial \ddot{\theta}_{1}} \approx \frac{\Delta M_{1}}{\Delta \ddot{\theta}_{1}}=\frac{M_{1}^{n+1, k=k^{\prime}+1}-M_{1}^{n+1, k=k^{\prime}}}{\ddot{\theta}_{1}^{n+1, k=k^{\prime}+1}-\ddot{\theta}_{1}^{n+1, k=k^{\prime}}}=\frac{M_{1}^{n+1, k=k^{\prime}+1}-M_{1}^{n+1, k=k^{\prime}}}{\delta} \\
& \frac{\partial M_{1}}{\partial \ddot{\theta}_{2}} \approx \frac{\Delta M_{1}}{\Delta \ddot{\theta}_{2}}=\frac{M_{1}^{n+1, k=k^{\prime}+2}-M_{1}^{n+1, k=k^{\prime}}}{\ddot{\theta}_{2}^{n+1, k=k^{\prime}+2}-\ddot{\theta}_{2}^{n+1, k=k^{\prime}}}=\frac{M_{1}^{n+1, k=k^{\prime}+2}-M_{1}^{n+1, k=k^{\prime}}}{\delta} \\
& \frac{\partial M_{2}}{\partial \ddot{\theta}_{1}} \approx \frac{\Delta M_{2}}{\Delta \ddot{\theta}_{1}}=\frac{M_{2}^{n+1, k=k^{\prime}+1}-M_{2}^{n+1, k=k^{\prime}}}{\ddot{\theta}_{1}^{n+1, k=k^{\prime}+1}-\ddot{\theta}_{1}^{n+1, k=k^{\prime}}}=\frac{M_{2}^{n+1, k=k^{\prime}+1}-M_{2}^{n+1, k=k^{\prime}}}{\delta} \\
& \frac{\partial M_{2}}{\partial \ddot{\theta}_{2}} \approx \frac{\Delta M_{2}}{\Delta \ddot{\theta}_{2}}=\frac{M_{2}^{n+1, k=k^{\prime}+2}-M_{2}^{n+1, k=k^{\prime}}}{\ddot{\theta}_{2}^{n+1, k=k^{\prime}+2}-\ddot{\theta}_{2}^{n+1, k=k^{\prime}}}=\frac{M_{2}^{n+1, k=k^{\prime}+2}-M_{2}^{n+1, k=k^{\prime}}}{\delta}
\end{aligned}
$$

For the following subiterations $\left(k \geq k^{\prime}+3\right)$, the angular accelerations can now be calculated by equation (6) until the FSI convergence criterion is satisfied. For each leaflet $i$, this FSI convergence criterion is given by:

$$
\left|M_{i}^{n+1, k}-I_{i} \cdot \ddot{\theta}_{i}^{n+1, k}\right|<\varepsilon
$$

The convergence threshold $\varepsilon$ is set at one percent of $M_{i}^{n+1, k}$ :

$$
\varepsilon=\frac{M_{i}^{n+1, k}}{100}
$$

When the FSI convergence criterion is satisfied, the geometry and data of the time step are stored and the next time step is initiated.

From the angular acceleration obtained from equation (6), the angular velocity and position of each leaflet on the time level $t+\Delta t$ is calculated using a time-integration scheme. Because the flow solver uses a backward Euler scheme, this scheme is also preferred for the structural solver, as shown in [2],

$$
\left\{\begin{array} { l } 
{ \ddot { \theta } _ { i } ^ { n + 1 , k + 1 } = \frac { \dot { \theta } _ { i } ^ { n + 1 , k + 1 } - \dot { \theta } _ { i } ^ { n } } { \Delta t } } \\
{ \dot { \theta } _ { i } ^ { n + 1 , k + 1 } = \frac { \theta _ { i } ^ { n + 1 , k + 1 } - \theta _ { i } ^ { n } } { \Delta t } }
\end{array} \Rightarrow \left\{\begin{array}{l}
\dot{\theta}_{i}^{n+1, k+1}=\dot{\theta}_{i}^{n}+\Delta t \cdot \ddot{\theta}_{i}^{n+1, k+1} \\
\theta_{i}^{n+1, k+1}=\theta_{i}^{n}+\Delta t \cdot \dot{\theta}_{i}^{n}+\Delta t^{2} \cdot \ddot{\theta}_{i}^{n+1, k+1}
\end{array}\right.\right.
$$

\subsection{Adaptive time step size}

The maximum allowable time step size is restricted by the maximum allowable mesh motion, and thus by grid characteristics. A criterion based on the maximum leaflet movement can be posted:

$$
\dot{\theta}_{i}^{n} \cdot \Delta t^{n+1} \leq \Delta \theta_{\max } \quad \Rightarrow \quad \Delta t^{n+1} \leq \frac{\Delta \theta_{\max }}{\dot{\theta}_{i}^{n}}
$$

\subsection{Quadratic extrapolations from previous time steps}

When advancing to a new time step, the angular accelerations for the first subiteration $(k=0)$ are estimated based on a quadratic extrapolation from previous time steps. This prediction of the initial values results in faster convergence.

Also, instead of calculating the Jacobian every time step, the Jacobian is quadratically extrapolated from previous time steps and used in the subiterations $k \geq 1$. Thus, the recalculation of the Jacobian is only done when needed. This recalculation is triggered when the residual drop of our FSI convergence criterion becomes too small. In our case, an arbitrary criterion of one order of magnitude in residual drop between two subsequent subiterations is set. This can be summarized as follows: 
- $k=0$ : extrapolation of angular accelerations and check FSI convergence criterion (equation (10));

- $k \geq 1$ : angular accelerations from equation (6) with extrapolated Jacobian and check FSI convergence criterion (equation (10)), until

$$
\left|M_{i}^{n+1, k}-I_{i} \cdot \ddot{\theta}_{i}^{n+1, k}\right|>\frac{\left|M_{i}^{n+1, k-1}-I_{i} \cdot \ddot{\theta}_{i}^{n+1, k-1}\right|}{10}
$$

from then on, we set $k^{\prime}=k$ and perform two subiterations to recalculate the Jacobian:

- $k=k^{\prime}+1$ : perturbate angular acceleration of leaflet 1 with $\delta$;

- $k=k^{\prime}+2$ : perturbate angular acceleration of leaflet 2 with $\delta$;

- $k \geq k^{\prime}+3$ : angular accelerations from equation (6) with the newly calculated Jacobian and check FSI convergence criterion (equation (10)).

\subsection{Behavior in fully opened and fully closed position}

The angular position of a leaflet is restricted by a minimum and a maximum angle, respectively the fully closed and fully opened position. In reality, this is usually done by a physical blocking mechanism incorporated in the design of the hinges.

Numerically, these limitations can be modelled by setting the position equal to the restriction and recalculating the angular velocity and acceleration. This is implemented as follows. When the calculated position of a leaflet exceeds its limitations (i.e. $\theta_{i}^{n+1, k+1}<\theta_{\min }$ or $\theta_{i}^{n+1, k+1}>\theta_{\max }$ ), then the position is set equal to this limit (i.e. $\theta_{i}^{n+1, k+1}=\theta_{\text {min }}$ or $\theta_{i}^{n+1, k+1}=\theta_{\max }$ ). In order to move exactly to the limited position in the time step, the angular velocity $\dot{\theta}_{i}^{n+1, k+1}$ and acceleration $\ddot{\theta}_{i}^{n+1, k+1}$ are subsequently recalculated (using the backward Euler scheme):

$$
\begin{aligned}
& \theta_{i}^{n+1, k+1}=\theta_{\min } \text { or } \theta_{\max } \\
& \dot{\theta}_{i}^{n+1, k+1}=\frac{\theta_{i}^{n+1, k+1}-\theta_{i}^{n}}{\Delta t} \\
& \ddot{\theta}_{i}^{n+1, k+1}=\frac{\dot{\theta}_{i}^{n+1, k+1}-\dot{\theta}_{i}^{n}}{\Delta t}
\end{aligned}
$$

However, the previously described FSI convergence criterion must be changed when the position is limited. The FSI convergence criterion should no longer depend on the absolute value of $M_{i}^{n+1, k}-I_{i} \cdot \ddot{\theta}_{i}^{n+1, k}$, but merely on its sign. This can be understood by adding $M_{i}^{r}$ to the angular momentum equation, with $M_{i}^{r}$ being the reaction moment of the blocking mechanism exerted on the leaflets:

or

$$
M_{i}^{r}+M_{i}^{n+1, k}=I_{i} \cdot \ddot{\theta}_{i}^{n+1, k}
$$

$$
M_{i}^{r}=-\left(M_{i}^{n+1, k}-I_{i} \cdot \ddot{\theta}_{i}^{n+1, k}\right)
$$

In order to maintain contact between the leaflet and the blocking mechanism, this reaction moment will be either negative or positive (depending on the case: $\theta_{\min }$ or $\theta_{\max }$ ). As FSI convergence criterion, it is checked whether or not the reaction moment has this appropriate sign. So when the motion of leaflet $i$ is limited, the FSI convergence criterion (equation (10)) of this leaflet $i$ is replaced by

$$
M_{i}^{r}<0
$$

or

$$
M_{i}^{r}>0
$$

depending on the orientation of the leaflet and on whether the leaflet is fully opened or fully closed. 


\section{Numerical simulation of a 3D BMHV}

The described algorithm is implemented in FLUENT to simulate a 3D case. The used BMHV is a simplified model of the ATS Open Pivot Standard Heart Valve with the size of $25 \mathrm{~mm}$ in aortic position. The orifice inner diameter measures $20.8 \mathrm{~mm}$. Furthermore, the $3 \mathrm{D}$ geometry consists of a straight rigid tube upstream of the valve, and rigid Valsalva sinuses downstream of the valve, as visualized in figure 3. The Valsalva sinuses are based on the geometry described in [5] and positioned asymmetrically with respect to the leaflets. It is expected that this asymmetrical geometry induces an asymmetric flow field downstream of the valve, resulting in an asynchronous motion of the valve leaflets. Therefore, it is an effective test for our algorithm with two degrees of freedom.

An unstructured grid is generated in the geometry and the arbitrary Lagrangian-Eulerian (ALE) approach is followed, which implies that the grid follows the motion of the structure and therefore needs an update. If applicable, this update is done using dynamic remeshing and spring smoothing.

An inlet aortic flow pulse with a time cycle of 1 s (displayed in figure 2) is imposed upstream. Since in a rigid tube geometry the pressure level does not affect the flow field (only the pressure gradient appears in the equation), a constant pressure is imposed at the downstream outlet boundary. Blood is modelled as a laminar incompressible fluid and a no-slip condition is applied at the walls. The valve is initially set in the closed position.

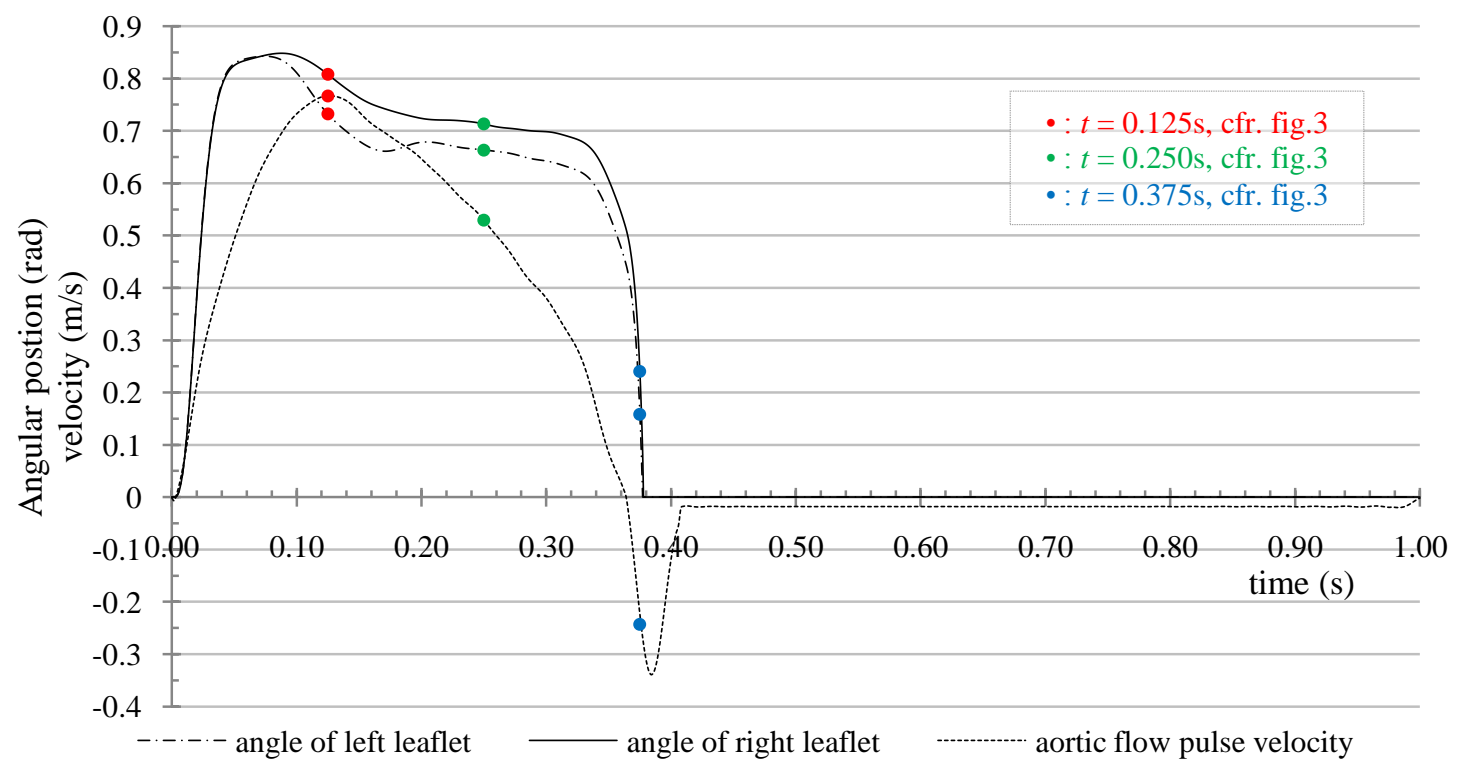

Figure 2. Angular position of leaflets and aortic flow pulse velocity.

The results of the simulation are depicted in figure 3, which shows the velocity magnitudes and static pressure contours. Figure 2 presents the angular positions of the leaflets, in which 0 rad and $1 \mathrm{rad}$ respectively refers to the fully closed and fully opened position. However, the results show that this maximum opening angle is not reached. Such incomplete opening is typical for the ATS Open Pivot valve and is described in literature. In [6], this phenomenon was explained due to the greater sensitivity of the leaflet movement to the flow field compared to other BMHV designs, since the leaflets extend farther in the flow downstream of the orifice than is the case in other designs. Also, it is found that the two leaflets show differences in movement. As mentioned above, it can be understood that this asynchrony is triggered by the presence of the asymmetric Valsalva sinuses geometry downstream of the valve. 

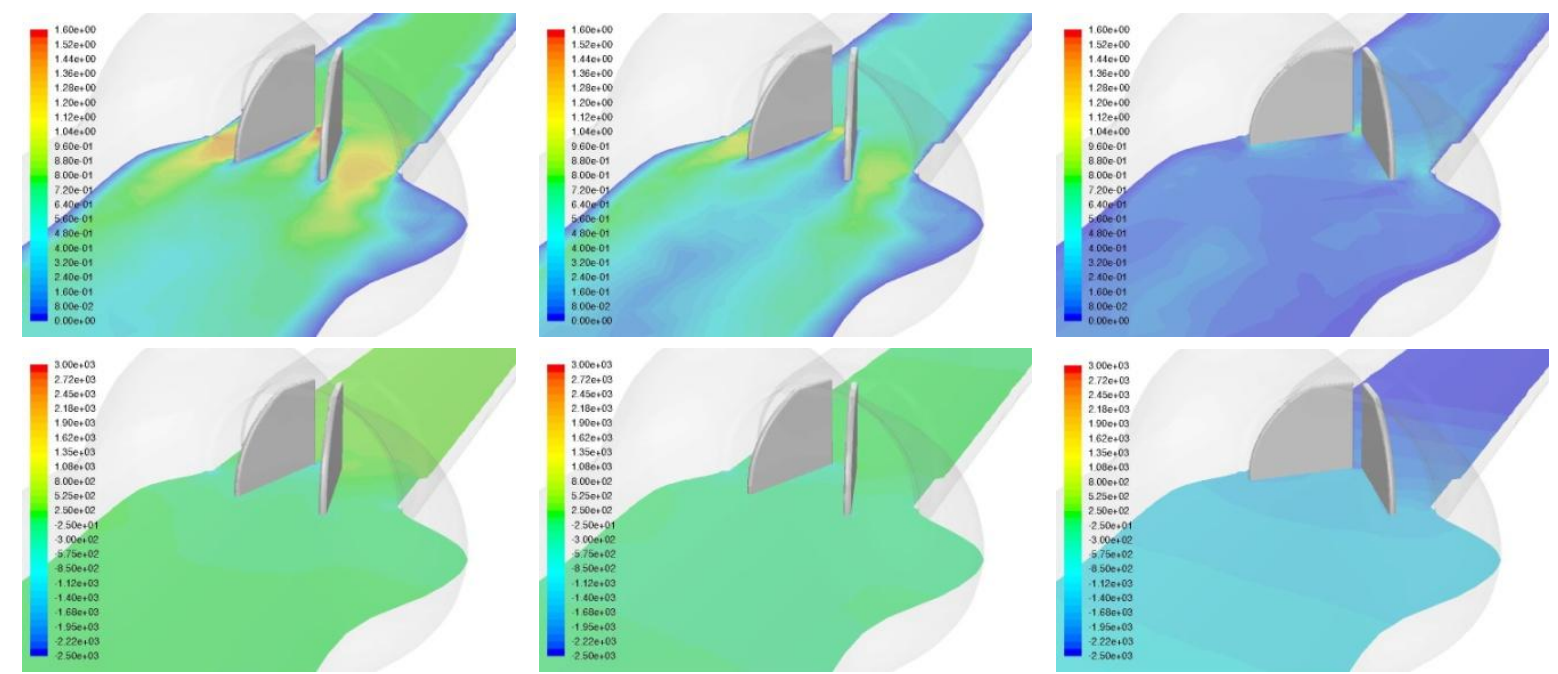

Figure 3. Velocity Magnitude Contours in $\mathrm{m} / \mathrm{s}(u p)$ and Static Pressure Contours in Pa (down), visualized on a longitudinal section perpendicular to the leaflet rotation axes, at $t=0.125 \mathrm{~s}$ (left), $t=0.250 \mathrm{~s}$ (middle) and $t=0.375 \mathrm{~s}($ right), respectively the red, green and blue dot in figure 2 .

\section{Comparison with existing coupling schemes}

The introduction of a subiteration loop within each time step implies the need for a stable and efficient approximation of the subsequent angular acceleration $\ddot{\theta}_{i}^{n+1, k+1}$ in each subiteration $k$, as mentioned above. Such approximation is usually achieved through a relaxation scheme:

$$
\ddot{\theta}_{i}^{n+1, k+1}=\ddot{\theta}_{i}^{n+1, k}+\omega_{i}^{n+1, k} \cdot\left(\frac{M_{i}^{n+1, k}}{I_{i}}-\ddot{\theta}_{i}^{n+1, k}\right)
$$

This can be rewritten as

$$
\ddot{\theta}_{i}^{n+1, k+1}=\left(1-\omega_{i}^{n+1, k}\right) \cdot \ddot{\theta}_{i}^{n+1, k}+\omega_{i}^{n+1, k} \cdot \frac{M_{i}^{n+1, k}}{I_{i}}
$$

If chosen properly, the relaxation factor $\omega_{i}^{n+1, k}$ stabilizes the solution process of the subiterations as described in [3].

For the simulation of a BMHV in a partitioned way, several methods can be used to find such an appropriate relaxation factor. We will discuss the use of a "fixed" relaxation factor and a "dynamic" relaxation factor. In the "fixed" case, the factor is kept at a constant value during the entire time cycle, as described in [7] for an industrial shock absorber valve. For the dynamically changing relaxation factor, a best fitted factor value is obtained in each time step. Typically, the Aitken $\Delta^{2}$ relaxation is used, as applied in [3]. This Aitken factor is in each subiteration $k>1$ given by

$$
\omega_{i}^{n+1, k}=\omega^{n+1, k}=-\frac{\left([\ddot{\theta}]^{n+1, k}-[\ddot{\theta}]^{n+1, k-1}\right)^{T}\left([r]^{n+1, k}-[r]^{n+1, k-1}\right)}{\left([r]^{n+1, k}-[r]^{n+1, k-1}\right)^{T}\left([r]^{n+1, k}-[r]^{n+1, k-1}\right)}
$$

with

$$
[\ddot{\theta}]^{n+1, k}=\left[\begin{array}{c}
\ddot{\theta}_{1}^{n+1, k} \\
\ddot{\theta}_{2}^{n+1, k}
\end{array}\right] \text { and }[r]^{n+1, k}=\left[\begin{array}{l}
\frac{M_{1}^{n+1, k}}{I_{1}}-\ddot{\theta}_{1}^{n+1, k} \\
\frac{M_{2}^{n+1, k}}{I_{2}}-\ddot{\theta}_{2}^{n+1, k}
\end{array}\right]
$$


The two techniques are implemented in FLUENT and used to simulate the same 3D case as in section 3. This allows a comparison of their stability and efficiency through numerical experiments. Simulations are conducted for different values of the fixed relaxation factor, for example $0.05,0.15$, 0.25 and 0.285 , resulting in resp. 17, 3, 20 and 107 subiterations for the first time step in the fully closed position. Thus, the value of 0.15 can be seen as the optimum, as graphically shown in figure 4 . However, with this optimal value for the first time step, approximately 5 subiterations per time step are needed to achieve convergence as soon as the valve is a few degrees opened, leading to 11 subiterations for the almost fully opened case. When using Aitken, a relaxation factor of 0.149 was also found for the first time step. However, in the following time steps, the Aitken relaxation factor rises steeply until (after approximately 80 time steps with constant $\Delta t=0.00025 \mathrm{~s}$ ) a stable value of approximately 0.375 is obtained, achieving convergence within 3 to 4 subiterations during valve opening, as depicted in figure 5. However, when setting the fixed relaxation factor to this optimal value of 0.375 for the entire time cycle, this leads to divergence in the first time step. Therefore, the results indicate that the dynamic relaxation technique outperforms the fixed relaxation. This was expected because the fixed factor is a trade-off value that needs to allow stability throughout the entire time cycle, in contrast to the dynamic factor which can be optimized for each specific time step (and subiteration) of the time cycle. We note that the value of our stable Aitken relaxation factor, i.e. approximately 0.375 , is of the same magnitude as the Aitken relaxation factors computed in [3].

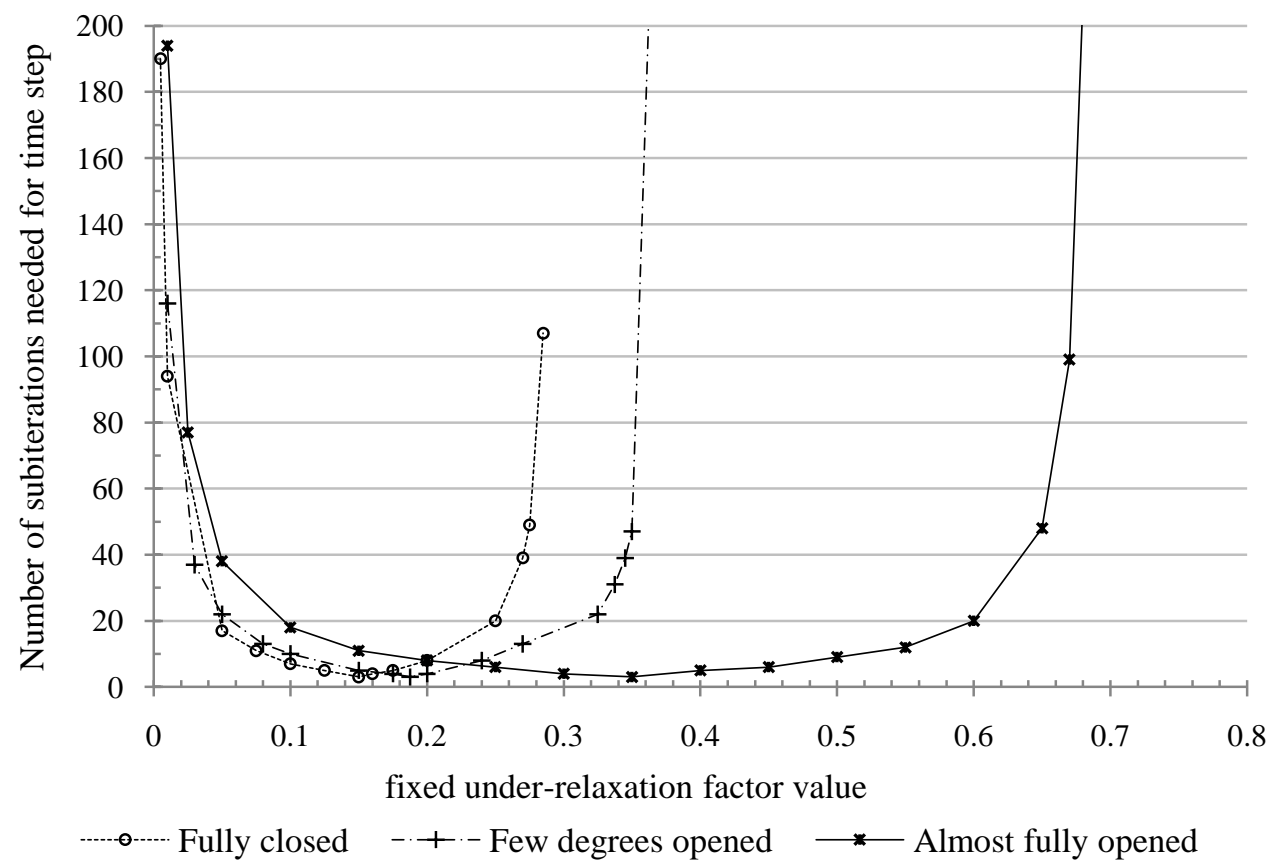

Figure 4. Influence of the fixed relaxation value.

Finally, the dynamically varying techniques are compared with each other. The opening phase of the valve, from $t=0 \mathrm{~s}$ to $t=0.125 \mathrm{~s}$ (peak systole), is simulated using 500 time steps with constant time step size. On average, Aitken needs 3.2 subiterations per time step, while convergence is achieved in 2.7 subiterations per time step when using our new method which uses linearization through the Jacobian. Thus, during the opening phase of the valve, Aitken $\Delta^{2}$ relaxation is found more expensive than the new method using the linearized approximation with the Jacobian. 


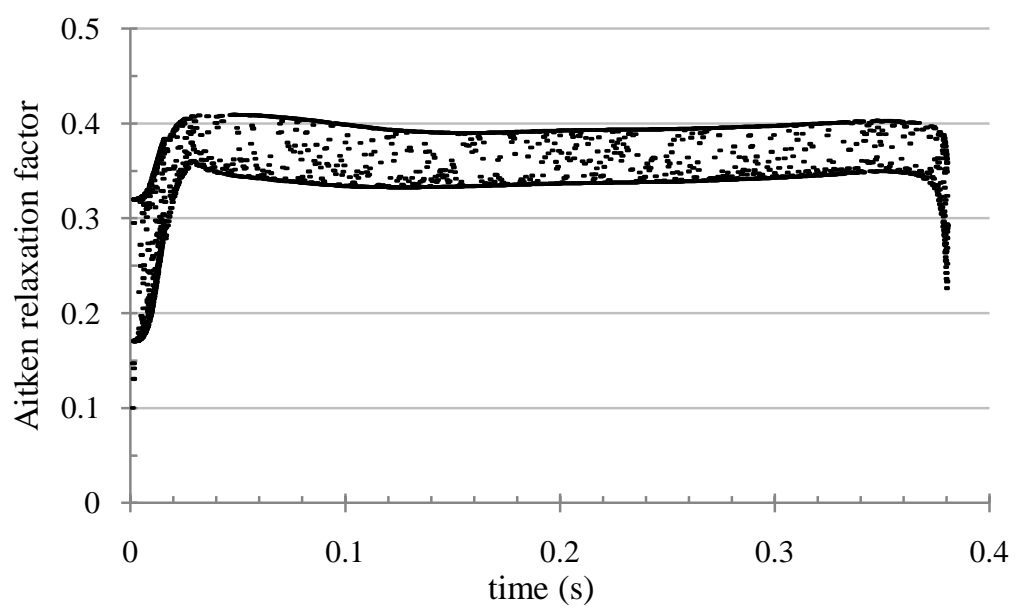

Figure 5. Range of the used Aitken relaxation factor values as a function of the time.

\section{Conclusions}

A new implicit FSI coupling algorithm for the partitioned simulation of a BMHV was presented. The algorithm was successfully tested and implemented for a 3D case.

A comparison was made with two existing coupling schemes. It was found that our newly developed FSI coupling scheme outperforms these coupling schemes.

\section{Acknowledgements}

Sebastiaan Annerel is funded by a BOF-grant (Special Research Fund) from Ghent University Association. Joris Degroote gratefully acknowledges a Ph.D. fellowship of the Research Foundation Flanders (FWO).

\section{References}

[1] Sotiropoulos F and Borazjani I 2009 A review of state-of-the-art numerical methods for simulating flow through mechanical heart valves Med. Biol. Eng. Comput. 47 245-56

[2] Vierendeels J, Dumont K, Dick E and Verdonck P 2005 Analysis and Stabilization of FluidStructure Interaction Algorithm for Rigid-Body Motion AIAA J. 43 2549-57

[3] Borazjani I, Ge L, and Sotiropoulos F 2008 Curvilinear immersed boundary method for simulating fluid structure interaction with complex 3D rigid bodies J. Comput. Phys. 227 7587-620

[4] Dumont K, Vierendeels J, Kaminsky R, van Nooten G, Verdonck P and Bluestein D 2007 Comparison of the Hemodynamic and Thrombogenic Performance of Two Bileaflet Mechanical Heart Valves Using a CFD/FSI Model J. Biomech. Engrg. 129 558-65

[5] Reul H, Vahlbruch A, Giersiepen M, Schmitz-Rode Th, Hirtz V and Effert S 1990 The geometry of the aortic root in health, at valve disease and after valve replacement $J$. Biomech. 23 181-91

[6] Feng Z, Umezu M, Fujimoto T, Tsukahara T, Nurishi M, Kawaguchi D and Masuda S 1999 Analysis of ATS leaflet behaviour by in vitro experiment $J$. Artif. Organs 2 46-52

[7] Le Tallec P and Mouro J 2001 Fluid structure interaction with large structural displacements Comput. Methods Appl. Mech. Engrg. 190 3039-67 